\title{
VULNERABILIDAD Y VIOLENCIA EN LA INFANCIA.
}

\author{
SILVIA GÓMEZ SOLDEVILLA \\ ÀNGELS RENON SOTORRA \\ CRISTINA RIMBAU ANDREU \\ E.U. Trabajo Social. Universidad de Barcelona.
}

\section{PRESENTACION.}

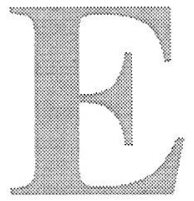

I proyecto EVE (Etude vulnerabilité Enfance) realizado en el marco de los Proyectos Europeos Daphne, en cinco países europeos: Bélgica, Francia, Luxemburgo, Grecia y España, en el período 2000-2001, centró su objetivo en el análisis de los factores de vulnerabilidad y violencia en los escenarios de la escolaridad primaria de los diferentes países participantes.

A continuación, se presenta, en primer lugar, la metodología utilizada y, en segundo lugar, los resultados obtenidos. Se trata de una investigación de carácter cualitativo y experimental, centrada en el análisis de las relaciones personales y de las redes de relación social como factores influyentes y de referencia para potenciar y trabajar situaciones de vulnerabilidad y violencia en la infancia-adolescencia. Se establecieron tres ejes relacionales de análisis:

- Entre los escolares y los adultos presentes en el ámbito escolar como elementos referenciales educativos, es decir, los profesores de todas las materias, el personal no docente, el personal de gestión y dirección, así como las asociaciones de madres y padres.

- Entre los escolares y la escuela y los servicios sociales, sanitarios, de justicia, policía, cívico-culturales, del barrio de ubicación de la escuela.

- Entre los escolares y sus familiares.

Podemos avanzar como resultados que, en los cinco países, se detectó que existía a la par una doble situación: por un lado la manifestación de un alto interés y reconocimiento por parte de todos los adultos del rol educativo que las instituciones escolar, familiar, y social desempeñan en la socialización y prevención de la violencia, y por otra el reconocimiento de que todas ellas cumplen limitadamente este rol. 


\section{METODOLOGÍA.}

En primer lugar, se delimitó el ámbito de estudio: una escuela pública de cada uno de los países participantes, ubicada en la trama urbana de una gran ciudad, de un barrio de clase media baja, con índices de inmigración importantes. El intervalo de edad de los alumnos en todos los países fue de niños de 8 a 12 años.

En segundo lugar, se estableció la metodología de contacto y entrada en las escuelas. Este punto fue de gran interés, puesto que el escenario de estudio era la propia escuela y las relaciones que se establecían dentro y fuera de ella. Cabe decir que en dos países representó un escollo importante debido a las resistencias (formales e informales) presentadas por profesores o la parte directiva, o por conflictos que se estaban produciendo en el ámbito escolar, como, por ejemplo, las huelgas de alumnos en Francia.

Los instrumentos de trabajo en las escuelas y con los servicios sociales y sanitarios externos, pero relacionados con ellas, fueron los siguientes:

- Con los alumnos. Se realizaron tres tipos de aproximación complementarias: 1) El pase de un cuestionario elaborado y validado previamente por la Universidad de Mons, y que fue previamente adaptado linguiísstica y culturalmente en cada uno de los países. El número total de cuestionarios realizados fue de

2) Realización de grupos de palabra para cada uno de los niveles de edad de los alumnos en cada una de las escuelas. En cada escuela se celebraron de cinco a seis grupos de palabra. 3) Observación pautada en espacios comunes de la escuela, tales como patio, comedor, entradas, pasillos, etc.

- Con los maestros de las escuelas. Se realizaron sesiones de trabajo en pequeños grupos de cuatro profesores, para recoger su percepción, opinión, valoración y propuestas sobre la vulnerabilidad y violencia en la escuela. Estos grupos eran voluntarios y tuvieron un elevado índice de participación. Para establecer dichos grupos se realizaron reuniones previas con los claustros de profesores. Así mismo, se consultó previamente con los claustros de cada escuela antes de presentar el estudio a los alumnos y la realización de los cuestionarios y los grupos de trabajo citados.

La participación del profesorado fue un elemento clave en el desarrollo de toda la investigación, no sólo para establecer las reuniones de análisis con ellos, sino también para crear un clima propicio para la colaboración de alumnos, familiares y otros profesionales vinculados con la escuela. De tal manera que, en un país, las barreras iniciales de los maestros pusieron en peligro la realización de la 
investigación.

- Con otros profesionales de las escuelas. Este grupo fue voluntariamente incorporado como un elemento muy importante en la investigación por su rol educativo informal en el ámbito escolar. El diseño de la investigación partía de la base de la institución escuela como un "todo educativo" en el cual todos los elementos desempeñan una función influyente (no neutra) en las relaciones de tolerancia y/o violencia del entorno. Efectivamente este colectivo se rebeló como clave en todas las escuelas de los diferentes países, tanto por su rol de observación y conocimiento de las relaciones en situaciones "informales" y aparentemente "aeducativas", como por el valor de sus actitudes e intervenciones en la prevención, educación o mediación de situaciones conflictivas. Así, se puso de manifiesto en todos los países el poder de su rol educativo.

El trabajo con este grupo de profesiones se desarrolló a partir de pequeños grupos de palabra. Debemos señalar que en un primer momento los propios profesionales dudaban del valor de su participación en una investigación, proyectando así el valor social que la "institución escuela" otorga a estos perfiles profesionales. Sin embargo, sus aportaciones, salvando las diferencias léxicas, se aproximaban y complementaban con las de los profesionales enseñantes. Es decir, el rol informal tenía un valor limitado o mínimo al inicio, pero se manifestó muy importante a lo largo de la investigación.

- Con los padres. La aproximación a los familiares de los alumnos se realizó de manera distinta en los diferentes países, respetando los diferentes sistemas de participación de éstos en las escuelas y también las "formas culturales" de relación entre los responsables de los alumnos y la institución escolar.

Metodológicamente, en Francia y España, se trabajó mediante grupos de madres-padres participantes regularmente en el ámbito escolar. Ello supone un cierto sesgo, pero también la posibilidad de trabajar este tema. Una aproximación más amplia podría haber dado una perspectiva mayor, pero también podría haber frustrado las posibilidades de análisis en el marco establecido en este trabajo.

En este punto, debe constatarse una fuerte feminización de la participación parental en las escuelas. Así, por ejemplo, en el grupo de la escuela del área metropolitana de Barcelona, si bien la asociación y la junta directiva están formadas por un cierto equilibrio por madres y padres, en las reuniones realizadas, el número y rol activo de las madres fue extremadamente superior al de los padres (se había realizado consulta y control previo de horarios de preferencia).

- Con los servicios sociales, sanitarios, culturales, etc. en relación con las escuelas. Estos servicios constituyen los núcleos de la red 
externa de relación de las escuelas, principalmente interpretando que todos ellos, incluida la institución escolar, son dispositivos sociales en un territorio, y elementos de referencia para niños, padres, y ciudadanos en general.

En cada país se seleccionó cuáles eran los servicios de conexión con las instituciones escolares, tanto las que mantenían un vínculo oficial, y en muchas ocasiones de obligación, como es el caso, por ejemplo, de los servicios socio-sanitarios, como las entidades y asociaciones presentes en la misma área geográfica de la escuela y que mantenían relaciones con ella. Como, por ejemplo, asociaciones de padres de personas mayores o culturales y deportivas.

Metodológicamente se trabajó con entrevistas pautadas y abiertas realizadas a nivel individual con profesionales y/o responsables de dichos servicios. El objetivo fue siempre el análisis de su rol en la prevención y atención de las situaciones conflictivas en el ámbito escolar, que pueden potenciar vulnerabilidad y/o violencia individual o colectiva en la infancia-adolescencia.

Cabe señalar que las aportaciones de los profesionales de estos servicios fueron en general muy valiosas por su elevado nivel de elaboración teórica, principalmente en los servicios de salud mental, sanitarios, y en algunos casos de los servicios sociales comunitarios.

- Los seminarios de trabajo transnacionales fueron el otro eje metodológico del estudio. A lo largo del proyecto se realizaron cinco encuentros de trabajo correspondientes a las diferentes fases del proyecto. Estos seminarios no se limitaron a trabajar internamente los responsables del proyecto en cada uno de los países, sino que se abrieron a la participación de expertos en el tema en cada uno de los países, lo cual permitió incorporar un amplio conjunto de aportaciones teóricas al estudio.

- Metodológicamente el estudio finalizó con la presentación en cada uno de los países de los resultados globales y parciales del mismo a las instituciones participantes en el estudio, y a expertos y otras instituciones interesadas en el tema.

\section{LOS RESULTADOS.}

Los resultados de esta investigación son múltiples, tanto de tipo metodológico como de los derivados de los análisis realizados.

1. Queremos señalar, en primer lugar, que el estudio de la vulnerabilidad y la violencia desencadena en sí mismo un conjunto de actitudes de prevención, rechazo, negación, protección, etc., que deben 
contemplarse previamente en el planteamiento metodológico. Esta primera conclusión, esperada inicialmente, pone de manifiesto la necesidad de adecuar los procesos de aproximación y abordaje, previamente y de manera cualitativa, a todo planteamiento metodológico e instrumental.

En este aspecto se revelaron clave dos factores:

a) La necesidad de contar con elementos informales significativos que facilitasen el contacto con las personas y las instituciones, más allá de todos los procesos formales que lógicamente deben seguirse.

b) El tiempo. El valor temporal, ese bien escaso en nuestras sociedades, ha sido un elemento clave para poder establecer los vínculos y espacios profesionales necesarios para la investigación. En síntesis, podríamos decir que "dar tiempo", y "seguir el ritmo temporal" de las instituciones y de los sujetos implicados en la investigación, es básico para poder desarrollar una investigación en este tema. No se trata del tiempo de planificación de la investigación (ejercicio evidentemente necesario), sino de la adecuación del tiempo de la investigación con el tiempo de las entidades y población diana implicada.

2. En segundo lugar, constatar que estudios de este tipo permiten plantear en el interior de la institución escolar un tema latente pero en general no tratado, la vulnerabilidad y la violencia, y las percepciones a su alrededor. A partir de lo cual dichos estudios deben plantearse, a nuestro entender, desde una perspectiva altamente respetuosa y dotándose de elementos de atención a las dinámicas que desvelan. Se trata pues de plantear una investigación-acción que en este tema debe contemplar posibilidades de mediación y de contención a desarrollar por miembros propios o externos al equipo investigador.

3. A nivel transversal se ha constatado que la violencia y la vulnerabilidad son elementos consustanciales en la infancia y en la adolescencia, y que lo importante es trabajar en cómo "adquirir mecanismos de prevención frente a la vulnerabilidad en el proceso de desarrollo humano" y en cómo "enseñar a gestionar positivamente la energía violenta". Se trata pues de trabajar con la vulnerabilidad y la violencia y no solamente focalizar el trabajo en una teórica erradicación.

4. Se constató también que la institución-escuela es un dispositivo social pertinente, y en ocasiones óptimo, para observar, prevenir y educar estos temas. Pero constatar que ello significa un rol distinto de este dispositivo social, ampliando su rol actual caracterizado en todos los países con una función de aprendizajes instrumentales de 
diversas materias, hacia un trabajo de carácter psico-social, a nivel individual y colectivo.

5. La violencia y la fragilidad tienen también una dimensión institucional, la cual debe ser tratada como un elemento estructural desde el interior de la propia institución, la ausencia de análisis y tratamiento conlleva la proyección de dicha vulnerabilidad institucional a sus miembros individuales (adultos o niñós).

6. Se constata también el importante rol de los adultos como tales, como elementos referenciales para los niños, tanto si se trataba de los profesores encargados de diferentes materias, como de los adultos responsables de tareas de vigilancia y orden en la escuela. Desde este punto de vista, en todos los países se evidenció el infravalor dado a estos perfiles profesionales y su casi ausencia o exclusión de los foros pedagógicos y de coordinación de las instituciones escolares, a pesar de que en algunos países se contempla su participación formal en órganos de gestión interna.

7. Los espacios informales de relación, más permisivos, más libres, para establecer relaciones y códigos de relaciones, emergieron con fuerza como espacios privilegiados en donde observar y trabajar las actitudes y relaciones entre niños y entre niños y adultos. En este sentido, la investigación insistió en sus conclusiones en la necesidad de incorporar estos espacios como espacios educativos, de amplia participación y dinámica. No se trata de convertir estos espacios en "alter egos" de los espacios clase, sino de incorporar su fuerza y posibilidades a la función educativa de la institución escolar tal como ya lo están realizando los propios alumnos y adultos.

8. Los dispositivos sociales, sanitarios, socio-pedagógicos de la red de relaciones externas y territoriales de la escuela, han sido considerados en todos los países como unos elementos referenciales de ayuda, soporte y orientación muy importantes, tanto para la institución escolar como por los miembros de las familias. Si bien se han detectado resistencias importantes de participación real por parte principalmente de las instituciones escolares, las cuales han manifestado en ocasiones cierta tendencia endógena de tratamiento y resolución de los conflictos. En cambio las experiencias de colaboración interinstitucional han sido valoradas en términos generales muy positivas y de ayuda.

9. Tanto los maestros, como los adultos de las escuelas, y los padres consultados coinciden en la necesidad de ayuda y formación en el tema de la violencia y la vulnerabilidad. Coinciden en que existe un nivel informativo bastante amplio, así como algunas instituciones sociales a las que recurrir en caso necesario, pero todos hacen hincapié en el cómo proceder y el cómo actuar. Se trata más de una 
demanda cualitativa de orientación y formación, que dé una demanda cuantitativa de mayores recursos o más información. En Bélgica, Francia y España, se puso de manifiesto también una cierta crítica a ciertos servicios externos que actuan más como receptores informativos que como elementos de ayuda y tratamiento del problema.

10. Finalmente indicar, como conclusión, que las aportaciones de los propios alumnos sobre el tema de la violencia y la vulnerabilidad fueron altamente cualitativas, mostrando un nivel de conocimiento e información del tema muy elevado, así como realizando sugerencias de cómo trabajar la violencia en la escuela. Sólo un ejemplo de los múltiples que podríamos citar. Preguntados sobre qué se podría hacer para disminuir o erradicar la violencia en la escuela y en la sociedad, nos ofrecieron las siguientes respuestas que correlacionan perfectamente con grandes líneas clásicas de las políticas sociales: - Hablar. "Hablar del tema en la escuela, en casa, en el patio, con alguien que pueda escucharnos y decirnos qué". Es decir, el silencio o la ignorancia institucional sobre el tema no ayuda, no resuelve.

- Encerrar, segregar. "Encerrar a todos los violentos, con más policías, más vigilancia, y que no salgan". Como ustedes pueden recordar ésta fue la línea de acción prioritaria de actuación en el siglo XVIII y XIX (el gran encierro) y ha continuado presente, con mayor o menor fuerza, hasta nuestros días.

- Ayudar. "Ayudar a las personas, los niños, que tienen problemas con las drogas o que son violentos". Al lado del control social ha existido también siempre la vertiente de asistencia y ayuda.

11. Por último, indicar que, desde el trabajo de campo y desde las aportaciones teóricas, se ha constatado también cómo la sociedad en general y en particular los núcleos familiares, están cambiando sus roles educativos con relación a las actitudes, valores personales y sociales, estructura interna, realizando un cierto desplazamiento de estos roles a instituciones formales externas, como pueden ser la escuela, servicios sociales, sanitarios, etc., y que ello está afectando al tratamiento y mediación de las situaciones de vulnerabilidad infantil y violencia.

\section{BIBLIOGRAFÍA.}

DEFRANCE, B.: “La Violence à l'école”. Syros/Alternatives, 1988.

GATTI, U.: "La devianza "nascota" dei giovani: una ricerca sugli studentidi tre città italiane". Rassegna italiana di criminologia: organo ufficiale dellasocietà italiana di criminologia, vol. 5, 1994, nº 2 . 
FERNÁNDEZ GARCÍA, I.: "Estudio y modelo de intervención de la violencia interpersonal en centros educativos". Bienestar y protección infantil, 1996, $\mathrm{n}^{\circ} 2$.

HELLMAN, D.A. y BEATON, S.: "The Pattern of violence in urban public schools: the influence of shool and community". Journal of research in crime and delinquency, 1986, $\mathrm{n}^{\circ} 2$.

La Inadaptación escolar grave en la segunda etapa de EGB: análisis de la situación, sugerencias y recomendación para la prevención. Pamplona: Fundación Bartolomé de Carranza, 1984.

Prevention de la délinquance juvénile, le role des institutions de socialisation dans une societé en évolution: rapports présentés a la quatorzième conference de recherches criminologiques. Strasboug: Conseil de l'Europe, 1982.

WALKER, H.M.; COLVIN, G.; RAMSEY, E.: Antisocial behavior in school: strategies and best practices. Pacific Grove: Brooks/Cole, 1995.

ORTEGA RUIZ, R. et al.: La Convivencia escolar: qué es y cómo abordarla. Sevilla: Junta de Andalucía. Consejería de Educación y Ciencia, DL, 1988.

FERNÁNDEZ, I.: "Prevención de la violencia y resolución de conflictos: el clima escolar como factor de calidad”. Educación hoy. Madrid: Narcea, DL, 1998.

CHARLOT, B.; ÉMIN, J.-C.: "Violences à l'école: état des savoirs". Formation des enseignants. Paris: Armand Colin, 1997.

HOFFMAN, A.M.: Schools, violence, and society. West Port, Conn.: Praeger, 1996.

MELERO MARTÍN, J.: "Conflictividad y violencia en los centros escolares". Psicología, etología y psicoanálisis. Madrid: Siglo XXI de España, 1993.

FUNES ARTIAGA, J.: Les aules taller $i$ els adolescents exclosos. Barcelona: Serveis de cultura popular, 1997.

PEÑA FANDIÑO, S.: Ponencia «Puntos de encuentro». $2^{\text {a }}$ Jornadas sobre Mediación y Servicios Comunitarios. Mollet, octubre 1998.

POURTOIS, J.P., DESMETH, H. et NIMAL, P. (2000): Vécu psychosocial et performance scolaire, à paraître.

Pourtois, J.P. et Bodson, V., (1999) Questionnaire-bilan des besoins, version enfant 9-13 ans édité par le CERIS de la FPSE de l'UMH.

POURTOIS, J.P. et DESMET, H. (1993): «L'éthique entre le savoir et la pratique» Ethica, vol. 5, No 1 .

DEBARBIEUX E., BLAYA, C.: Les parents, la violence, l'école: un détour européen. In Ville-Ecole-Intégration, No 114, septembre 1998.

GUIBERT, L.: Pour une éducation non-violente: développer l'agressivité des enfants! In Les Rencontres de Nantes. De. ACCOORD, Nantes.

PAIN, J.: Violences et prévention de la violence à l'école, In Les Sciences de l'éducation 30, 2. 1997.

SYNHESE: «Villence in schools: awareness-raising, prevention, penalties» Conseil de l'Europe, De. Council of Europe Publishing, Strasbourg, 1999. 